\title{
ПОЛИНОМИАЛЬНЫЙ АНАЛИЗ ТРЕНДА МОЩНОСТИ ЗАЛЕЖИ ДЛЯ ПОДСЧЕТА ЗАПАСОВ МЕСТОРОЖДЕНИЯ НА ЭВМ
}

В настоящее время использование математических методов и ЭВМ при обработке и анализе геологической информации возрастает быстрыми темпами. Одной из наиболее эффективных форм применения ЭВМ в геологии стали автоматизированные системы подсчета и оценки запасов полезных ископаемых. Такие системы ускоряют процедуру подсчета и оценки запасов, освобождают геологов от большого объема однообразной механической работы и, что особенно важно, позволяют производить комплексный анализ исходной информации с минимальными методическими упрощениями, неизбежными при анализе данных вручную или с помощью отдельных программ на ЭВМ. Последнее дает возможность более правильно оценивать перспективность месторождений и решать вопросы их комплексной разработки.

Хотя традиционные методы подсчета запасов (способы разрезов, многоугольников) выдержали проверку практикой - с их помощью успешно оценены запасы большого числа месторождений, необходимость в совершенствовании этих методов с развитием электронной вычислительной техники не вызывает сомнений. ЭВМ не только создает условия для выполнения большого объема вычислительных работ, но и при наличии готовой программы освобождает геологов от надобности овладевать техникой проведения вычислений.

При разработке способов оценки объема запасов задача сводится к нахождению по разведочным данным такой поверхности мощности залежи, которая бы наилучшим образом аппроксимировала реальные изменения мощности по площади. После того как эта поверхность найдена, вычисление объема не представляет трудностей.

Общепринятым способом построения поверхности по системе заданных на плоскости значений является аппроксимация поверхности степенными или тригонометрическими полиномами с нахождением ощенки их параметров по методу наименьших квадратов (Крамбейн, Грейбилл, 1969; Davis, 1973; Harbaugh, Merriam, 1968). Однако в приложении к реальным условиям разведки месторождений этот метод не обеспечивает удовлетворительной аппроксимации из-за появления ошибок округления при проведении вычислений с полиномами высоких порядков. Ниже излагается методика устранения ошибок такого рода с помощью центрирования исходных данных, приведения их к подходящему масштабу и применения корреляционной матрицы. номом

Исходная модель поверхности тренда мощности выражается поли- 


$$
P=\gamma_{0}+\gamma_{1} X+\gamma_{2} Y+\ldots+\gamma_{j} X q Y^{\prime}+\ldots+\gamma_{p} X^{t} Y^{t^{\prime}}+\varepsilon
$$

где $P$ - мощность залежи; $X, Y$ - георафические координаты пунктов исследования; $\gamma_{0}, \ldots, \gamma_{p}-$ параметры модели, подлежащие оцениванию; $\varepsilon-$ ошибка.

Процедуру начинают с центрирования исходных данных и приведения их к определенному масштабу с тем, чтобы уменьшить ошибки округления в последующих вычислениях. Появление таких ошибок связано с использованием слишком больших или слишком малых по абсолютной велнчине наблюдений при построении корреляционной матрицы. В результате полином (1) принимает вид

$$
p=\beta_{0}+\beta_{1} x+\beta_{2} y+\ldots+\beta_{j} x^{q} y^{q^{\prime}}+\ldots+\beta_{p} x^{t} y^{t^{\prime}}+\varepsilon,
$$

где $p=\frac{P-\bar{P}}{k_{p}}, x=\frac{X-\bar{X}}{k_{x}}, y=\frac{Y-\bar{Y}}{k_{y}}, \quad \beta_{0}, \ldots, \beta_{p}$ - параметры модели, подлежащие оцениваниг, $\bar{P}, \bar{X}, \bar{Y}-$ средние от переменных $P, X, Y$,

$k_{p}=\frac{\sum_{i=1}^{n}\left|P_{i}-\bar{P}\right|}{n}, \quad k_{x}=\frac{\sum_{i=1}^{n}\left|X_{i}-\bar{X}\right|}{n}, \quad k_{y}=\frac{\sum_{i=1}^{n}\left|Y_{i}-\bar{Y}\right|}{n} ; n-$ число наблюдений.

Далее модель (2) преобразуется к виду

$$
p=\beta_{0}+\beta_{1} Z_{1}+\beta_{2} Z_{2}+\ldots+\beta_{j} Z_{j}+\ldots+\beta_{p} Z_{p}+\varepsilon,
$$

где $Z_{j}=x^{q} y^{q}$, и затем записывается для удобства в матричной форме

$$
\vec{P}=\vec{Z} \vec{\beta}+\vec{\varepsilon}
$$

где $\vec{P}-(n \times 1)-$ вектор наблюдений $p ; \vec{\beta}-(p \times 1)-$ вектор параметров модели; $\vec{\varepsilon}-(n \times 1)$ - вектор ошибок;

$$
\vec{Z}=\left[\begin{array}{ccccccc}
1 & Z_{11} & Z_{21} & \ldots & Z_{j 1} & \ldots & Z_{p 1} \\
1 & Z_{12} & Z_{22} & \ldots & Z_{j 2} & \ldots & Z_{p 2} \\
1 & Z_{13} & Z_{23} & \ldots & Z_{j 3} & \ldots & Z_{p 3} \\
1 & Z_{1 i} & Z_{2 i} & \ldots & Z_{j i} & \ldots & Z_{p i} \\
1 & Z_{1 n} & Z_{2 n} & \ldots & Z_{j n} & \ldots & Z_{p n}
\end{array}\right] .
$$

и $Z_{j i}$ - наблюденное значение $Z_{j}$, которое соответствует наблюдению $p_{i}$ (Дрейпер, Смит, 1973).

Оценка для вектора $\vec{\beta}$, полученная по методу наименьших квадратов, есть векторная величина $\vec{b}$. Этот метод дает нормальные уравнения $\left(\vec{Z}^{\prime} \vec{Z}\right) \vec{b}=\overrightarrow{Z^{\prime} P}$. В нашей задаче с высокими порядками по $x, y$ и с большим объемом данных результаты, полученные непосредственно по формуле $\vec{b}=\left(\vec{Z}^{\prime} \vec{Z}\right)^{-1} \overrightarrow{Z^{\prime}} \vec{P}$, могут оказаться совершенно неверными по причине 
плохой обусловленности матрицы $\vec{Z} \cdot \vec{Z}$. Для улучшения метода вычислений $\vec{Z}^{\prime} \vec{Z}$ следует преобразовать в корреляционную матрицу (Дрейпер, Смит, 1973) с тем, чтобы все используемые в вычислениях числа оказались лежащими в интервале \pm 1 . В этом случае все числа будут одного порядка, что приведет к уменьшению ошибок округления.

Тогда нормальные уравнения для новой модели примут вид

$$
\left\{\begin{array}{ccccccc}
1 & r_{12} & r_{13} & \ldots & r_{1 j} & \ldots & r_{1 p} \\
r_{21} & 1 & r_{23} & \ldots & r_{2 j} & \ldots & r_{2 p} \\
r_{31} & r_{32} & 1 & \ldots & r_{3 j} & \ldots & r_{3 p} \\
\ldots & & & & \ldots & \\
r_{l 1} & r_{l 2} & r_{l 3} & \ldots & r_{l j} & \ldots & r_{l p} \\
\ldots & \ldots & & & & & \\
r_{p 1} & r_{p 2} & r_{p 3} & \ldots & r_{p j} & \ldots & 1
\end{array}|\times| \begin{array}{l}
a_{1} \\
a_{2} \\
a_{3}
\end{array}|=| \begin{array}{l}
r_{1 y} \\
r_{2 y} \\
r_{3 y} \\
a_{j} \\
r_{j y} \\
a_{p y}
\end{array}\right]
$$

где $a_{1}, \ldots, a_{p}$ - оценки параметров $\alpha_{1}, \ldots, \alpha_{p}$. Члены матрицы и векторы вычисляются по формулам

$$
r_{l j}=\frac{\sum_{i=1}^{n}\left(Z_{l i}-\bar{Z}_{l}\right)\left(Z_{j i}-\bar{Z}_{j}\right)}{\left[\sum_{i=1}^{n}\left(Z_{l i}-\bar{Z}_{l}\right)^{2} \sum_{i=1}^{n}\left(Z_{j i}-\bar{Z}_{j}\right)^{2}\right]^{1 / 2}}
$$

H

$$
r_{j y}=\frac{\sum_{i=1}^{n}\left(Z_{j i}-\bar{Z}_{j}\right) p_{i}}{\left[\sum_{i=1}^{n}\left(Z_{j i}-\bar{Z}_{j}\right)^{2} \sum_{i=1}^{n} p_{i}^{2}\right]^{1 / 2}}
$$

где $\bar{Z}_{j}=\frac{\sum_{i=1} Z_{j i}}{n}, j=1,2, \ldots, p$. Для получения средних и сумм, входящих в формулы (4) и (5), привлекаются рекуррентные уравнения (Neely, 1966):

$$
\bar{Z}_{j}^{(i)}=\frac{i-1}{i} \bar{Z}_{j}^{(i-1)}+\frac{1}{i} Z_{j}^{(i)}
$$

и

$$
Z_{l j}^{(i)}=Z_{l j}^{(i-1)}+\frac{i-1}{i}\left(Z_{l}^{(i)}-\bar{Z}_{l}^{(i-1)}\right)\left(Z_{j}^{(i)}-\bar{Z}_{j}^{(i-1)}\right),
$$

где $i=1, \ldots, n$ и $\bar{Z}_{j}^{(0)}=\bar{Z}_{l}^{(0)}=Z_{l j}^{(0)}=0$. Рекуррентный метод обладает повышенным быстродействием, поскольку каждое наблюдение участвует в обработке всего один раз.

Преобразования, сделанные выше для получения корреляционной матрицы из матрицы $Z^{\prime} Z$, включали репараметризацию. Оценки $b_{0}, b_{1}$, $b_{2}, \ldots, b_{p}$ промежуточных параметров $\beta_{0}, \beta_{1}, \beta_{2}, \ldots, \beta_{p}$ определяются из формул 


$$
b_{j}=a_{j} \frac{\sum_{i=1}^{n} p_{i}^{2}}{\sum_{i=1}^{n}\left(Z_{j i}-Z_{j}\right)^{2}}, j=1,2, \ldots, p
$$

и

$$
b_{0}=-b_{1} \bar{Z}_{1}-b_{2} \bar{Z}_{2}-\ldots-b_{j} \bar{Z}_{j}-\ldots-b_{p} \bar{Z}_{p},
$$

а оценки $c_{0}, c_{1}, c_{2}, \ldots, c_{p}$ исходных параметров $\gamma_{0}, \gamma_{1}, \gamma_{2}, \ldots, \gamma_{p}$ находятся путем замены переменных $p, x, y$ исходными обозначениями $\frac{P-\bar{P}}{k_{p}}, \frac{X-\bar{X}}{k_{x}}, \frac{Y-\bar{Y}}{k_{y}}$

в уравнении (2), путем раскрытия скобок, группирования параметров по степеням $X, Y$ и приведения подобных членов. Однако в случае предсказания значения мощности залежи необходимость в понске оценок исходных параметров отпадает, так как эти мощности можно определить по формуле

$$
\begin{gathered}
P=\bar{P}+k_{p}\left[b_{0}+b_{1} \frac{X-\bar{X}}{k_{x}}+b_{2} \frac{Y-\bar{Y}}{k_{y}}+\ldots\right. \\
\left.+b_{j}\left(\frac{X-\bar{X}}{k_{x}}\right)^{q}\left(\frac{Y-\bar{Y}}{k_{y}}\right)^{q^{\prime}}+\ldots+b_{p}\left(\frac{X-\bar{X}}{k_{x}}\right)^{t}\left(\frac{Y-\bar{Y}}{k_{y}}\right)^{t^{\prime}}\right] .
\end{gathered}
$$

Таким образом, полиномиальный анализ тренда является аналитическим методом, дающим в результате его применения уравнение поверхности тренда. Сечение такой поверхности параллельными плоскостями позволяет построить карту в виде изолиний. Ясно, что если при исследовании требуется выровнять данные карты для облегчения дальнейшей интерпретации лишь приблизительно, необходимость в трудоемкой процедуре вычисления поверхности тренда отпадает. Действительно, субъективный фактор играет в подобном исследовании весьма малую роль, поскольку поверхности тренда мало отличаются от поверхностей, получаемых путем графических построений (Harbaugh, Merriam, 1968). Если же такое уравнение необходимо для дальнейших исследований, применение полиномиального анализа тренда целесообразно. В нашем случае уравнение тренда предназначено для автоматизированной системы подсчета и оценки запасов. Кроме построения карт в изолиниях, с его помощью можно подсчитать запасы и даже организовать компактные информационно-поисковые системы непрерывной информации на магнитной ленте ЭВМ.

По изложенному алгоритму автором создана для ЭВМ *Минск-32» программа на языке ЯСК.

Экспериментальные исследования проведены по результатам разведки болотной системы Пухату в Северо-Восточной Эстонии, где на площади 55000 га представлены все типы торфяных залежей. Болотная система образовалась в послеледниковое время в результате заторфовывания мелководного озера.

Детальной разведкой изучен участок Ору площадью около 1000 га на северной оконечности месторождения. Здесь представлена только торфяная залежь низинного типа. Зондированием глубины, проведенным в 687 точках по сетке $150 \times 100$, установлено, что средняя мощность болотных отложений на участке составляет $3,7 \mathrm{M}$.

Результаты полиномиального анализа тренда мощности этих отложений в Ору (см. таблицу) показали, что точность аппроксимации 
вполне достаточна и для построения карт в виде изолиний и для подсчета запасов.

\begin{tabular}{l|c|c|c|c|c|c|c|c|c|c}
$\begin{array}{c}\text { Степень уравнения } \\
\begin{array}{c}\text { поверхности } \\
\text { тренда }\end{array}\end{array}$ & 1 & 2 & 3 & 4 & 5 & 6 & 7 & 8 & 9 & 10 \\
\hline $\begin{array}{l}\text { Коэффициент } \\
\text { корреляции }\end{array}$ & 0,70 & 0,86 & 0,90 & 0,92 & 0,92 & 0,94 & 0,95 & 0,95 & 0,96 & 0,97
\end{tabular}

Расчеты занимают при полиноме десятой степени и наличий 687 наблюдений 30 мин.

В настоящей статье построение математической модели рассмотрено на примере мощности залежи, но использовать ее можно и при описании качественных показателей залежи и вообще при оценке любых признаков в геологическом пространстве.

Автор выражает благодарность Э. Лелумеэсу и Я. Куксу за полезные замечания.

\section{ЛИТЕРА Т У Р А}

Д р ей п е р Н., С м и т Г. 1973. Прикладной регрессконный анализ. М., с. 150-159.

К р а м бе й н У., Г ре й билл Ф. 1969. Статистические модели в геологии. М., с. 251282.

Davis, I. S. 1973. Statistics and data analysis in geology. New York, p. 322-351.

$\mathrm{H}$ a rbaugh, J. W., Merriam, D. F. 1968. Computer applications in stratigraphic analysis. New York, p. 61-112.

Neely, P. M. 1966. Comparison of several algorithms for computation of means, standard deviations and correlation coefficients. Communs ACM, 9, No. 7, 496-499.

Ннститут геологии

Академии наук Эстонской ССР
Поступила в редакцию 29/III 1977

K. ORGLA

\section{MAARDLA LASUNDI PAKSUSE POLUNOOM-TRENDANALUUS VARUDE ARVESTAMISEKS ELEKTRONARVUTI ABIL}

Lasundi paksuse varieerumist kirjeldatakse matemaatilise mudeli - kolmemōōtmelise trendi vôrrandi abil. Mudeli koefitsiendid leitakse vähimruutude meetodil vôrrandist

$$
\vec{b}=(\vec{Z} \cdot \vec{Z})^{-1} \overrightarrow{Z^{\prime}} \vec{P}
$$

kus $\vec{b}$ on koefitsientide vektor, $\vec{Z}$ - sōltumatute muutujate (koordinaatide) maatriks ja $\vec{P}$ - sōltuva muutuja (paksus) vektor. Et laiendada meetodi kasutusala, vaatlusandmed tsentreeritakse ja mastabeeritakse; $\vec{Z} \vec{Z}^{\prime}$-maatriksi koostamisel kasutatakse rekurrentseid võrrandeid, kusjuures enne pööramist muudetakse $\overrightarrow{Z^{\prime} Z} \vec{Z}$ korrelatsioonimaatriksiks. Trendi vōrrandi abil vōib kirjeldada ka kvalitatiivsete näitajate muutumist lasundis. Otstarbekas on lülitada selline mudel lauglasumusega maavarade varude arvestamise ja hindamise automatiseeritud süsteemi.

Esitatud algoritmi järgi on arvutile «Minsk-32» koostatud SSK-keelne programm. 


\section{K. ORGLA}

\section{POLYNOMIAL TREND ANALYSIS OF THE THICKNESS OF DEPOSITS FOR CALCULATING RESOURCES BY COMPUTER}

The variation of a deposit in thickness is described by a mathematical model a three-dimensional trend equation. The coefficients of the equation are calculated by the least-squares criterion from the equation

$$
\vec{b}=\left(\overrightarrow{Z^{\prime} Z}\right)^{-1} \overrightarrow{Z^{\prime}} \vec{P}
$$

where $\vec{b}$ - the vector of coefficients, $\vec{Z}$ - the matrix of powers and products of independent variables (geographic coordinates), $\vec{P}$ - the vector of dependent variable (thickness).

Seeking to widen the realm of practical use of the method, observations are centered and scaled; for obtaining the entries in the matrix $\overrightarrow{Z^{\prime}} Z$, recurrent equations are used, and before inversion the matrix is converted into a correlation matrix.

The model described may also be used for a prediction of the qualitative variables of the deposit. It can be effectively used in the automatic system for calculating the resources and the quality of the deposits. The algorithm is programmed in a symbolic code language for the computer "Minsk-32".

\section{SISUKORD}

D. Ka $1 \mathrm{j}$ o. Daunton vôi Pridoli? (Balti siluri seisukohast). Resümee .

Viiu Nestor, $\mathrm{H}$, Nestor, M. Rubel. Costistricklandia lirata (Pentamerida, Brach.) Eesti ülemländouveris. Resümee

Tiiu Märss, R. E i n a s to. Siluri selgroogsete jaotumusest Pōhja-Balti basseini fatsiaalsetes setetes. Resümee

E. Pirrus, Leili $\mathrm{S}$ a a $\mathrm{r}$ s e. Lōuna-Eesti jääjărvesavide peene fraktsiooni struktuurilis-morfoloogilistest iseärasustest. Resümee

K. Orgla. Maardla lasundi paksuse polünoom-trendanalüüs varude arvestamiseks elektronarvuti abil. Resümee

\section{СОДЕРЖАНИЕ}

Д. К альо. Даунтон или пржндоли? (С точки зрения Балтийского силура). Резюме . . Вийу Н естор, Х. Несто р, М. Р убель. Costistricklandia lirata (Pentamerida, Brach.) из верхнего лландовери Эстонии

Тийу М я р с с, Р. Эй н асто. Распределение бертебрат в разнофациальных отложениях силура Северной Прибалтики

Э. П и р ру с, Лейли С а а р с е. К структурно-морфологической характернстнке тонкой фракции озерно-ледниковых глин Южной Эстонии

К. Оргла. Полиномиальный анализ тренда мощности залежи для подсчета запасов месторождения на ЭВМ

\section{CONTENTS}

D. Ka $\mathrm{j} \mathrm{jo}$. The Downtonian or Pridolian from the point of view of the Baltic Silurian . Viiu Nestor, H. Nestor, M. Ru bel. Costistricklandia lirata (Pentamerida, Brach.) in the Upper Llandovery of Estonia. Summary

Tiiu $M$ ärss, R. E in a sto. Distribution of vertebrates in deposits of various facies in the North Baltic Silurian. Summary

E. Pirrus, Leili $\mathrm{S}$ a a rse. Textural-morphological characterization of South Estonian limnoglacial clayey deposits. Summary

K. O r g l a. Polynomial trend analysis of the thickness of deposits for calculating resources by computer. Summary 